\title{
ENSEMBLE BASED ATMOSPHERIC PHASE SCREEN ESTIMATION USING LEAST SQUARES
}

\author{
Franz-Georg Ulmer, Nico Adam and Michael Eineder \\ DLR - Remote Sensing Technology Institute (IMF) \\ 82234 Wessling, Germany \\ Franz-Georg.Ulmer@dlr.de, Nico.Adam@dlr.de, Michael.Eineder@dlr.de
}

Copyright 2012 IEEE. Published in the IEEE 2012 International Geoscience \& Remote Sensing Symposium (IGARSS 2012), scheduled for July 22-27, 2012 in Munich, Germany. Personal use of this material is permitted. However, permission to reprint/republish this material for advertising or promotional purposes or for creating new collective works for resale or redistribution to servers or lists, or to reuse any copyrighted component of this work in other works, must be obtained from the IEEE. Contact: Manager, Copyrights and Permissions / IEEE Service Center / 445 Hoes Lane / P.O. Box 1331 / Piscataway, NJ 08855-1331, USA. Telephone: + Intl. 908-562-3966.

\begin{abstract}
It is a matter of fact that atmosphere causes a significant effect in interferometric measurements. A stack of acquisitions is needed to reduce this nuisance influence. Another approach is to use numerical weather predictions (NWP) [1]. However, it is not straight forward to choose parameters of a NWPsystem to get the best possible result. Also, different input data can be used.

Ensemble methods are well established to get a most reasonable forecast which is a mixture of differently initialized forecasts. Based on these weather predictions (NWPs), a collection of possible atmospheric phase screens (APSs) is computed and a most reasonable atmospheric phase screen (APS) is estimated. The idea behind this ensemble is, that the difference between every two pixels in an interferogram should match the difference of the corresponding pixels of a restricted linear combination of different estimated APS which results from different NWPs.

This paper describes the technique in detail and provides an example demonstration using real data.
\end{abstract}

Index Terms- InSAR, APS, NWP, PSI

\section{INTRODUCTION}

The main problems of NWPs are the uncertainties in the initial conditions and a very big pool of different options which can be chosen (e.g. resolution, size of simulated area, physics options, size of time steps). Another problematic issue is the timing in NWPs. In persistent scatterer interferometry (PSI), the atmospheric condition is needed at the time of the radar acquisition. Unfortunately the NWP model could have a delay, because of the necessary spin-up time.

The subject of the actual work is estimating the APS which best corresponds to the InSAR data from an ensemble of NWP generated APSs. This APS estimation will be modelled as a restricted linear combination of the different NWP based APSs.

To estimate the most plausible APS, a large restricted linear equation system is solved. Based on this set of equations, an argument minimum will be computed which comprises the most plausible restricted linear combination of simulated APSs.

The plausible APS estimation shall improve the precision in measuring deformations and therefore reduce the number of radar acquisitions which are needed and can be used for data assimilation for better weather forecasts as well.

\section{THEORY}

The phase $\Phi_{i, j}^{r}$ of a point scatterer at range-azimuth position $i, j$ of the real observed APS (superscript $r$ ) could be decomposed into [2]:

$$
\Phi_{i, j}^{r}=\Phi_{\mathrm{atmo}_{i, j}}^{r}+\Phi_{\text {defo } i, j_{i}}^{r}+\Phi_{\text {topo }_{i, j}}^{r}+\Phi_{\text {noise }_{i, j}}^{r}+\Phi_{\text {scat }_{i, j}}^{r}
$$

where $\Phi_{\text {atmo }_{i, j}}^{r}, \Phi_{\text {defo }_{i, j}}^{r}, \Phi_{\text {topo }_{i, j}}^{r}, \Phi_{\text {noise }_{i, j}}^{r}$ and $\Phi_{\text {scat }_{i, j}}^{r}$ are the corresponding phase delays caused by the atmosphere, the deformation, the topography, noise and object phase respectively.

An interferometric phase is defined by [2]:

$$
\Phi_{i, j}^{i n t_{r, r^{\prime}}}:=\Phi_{i, j}^{r}-\Phi_{i, j}^{r^{\prime}}
$$

In order to derive the framework we assume that the topography is perfectly compensated (by a very good available digital elevation model) and the deformation is much smaller than the atmosphere effect. Now, we can relate the real InSAR scatterer phase observation $\Phi_{i, j}^{r}$ to the $k$ 'th NWP APS ${ }_{k} \Phi_{i, j}^{s}$ 
(the superscript $s$ donates the NWP and the $r$ the InSAR data). Given $n$ NWPs for $s$ this relation is:

$$
\Phi_{i, j}^{r} \approx\left(\sum_{k}^{n} a_{k k}^{s} \Phi_{i, j}^{s}\right)+\Phi_{\text {scat }_{i, j}}^{r}
$$

while $a_{k}^{s}$ are not negative and $\sum a_{k}^{s} \approx 1$.

Practically, it is not possible to compute these $a_{k}^{s}$ directly, because the real state of the atmosphere is unknown. Well known is the phase difference between points in the interferogram, so following can be approximated:

$$
\begin{gathered}
\Phi_{i, j}^{i n t_{r, r^{\prime}}}-\Phi_{i^{\prime}, j^{\prime}}^{i n t_{, r^{\prime}}} \approx \\
\left(\sum_{k}^{n} a_{k}^{s}\left({ }_{k} \Phi_{i, j}^{s}-{ }_{k} \Phi_{i^{\prime}, j^{\prime}}^{s}\right)\right)-\left(\sum_{k}^{n^{\prime}} a_{k}^{s^{\prime}}\left({ }_{k} \Phi_{i, j}^{s^{\prime}}-{ }_{k} \Phi_{i^{\prime}, j^{\prime}}^{s^{\prime}}\right)\right) .
\end{gathered}
$$

It is obvious that a good approximation of (3) is also a good approximation of (4). The converse argument is not true in a mathematical meaning, but in reality it should hold. Because in reality all ${ }_{k} \Phi_{i, j}^{s}$ should be pretty similar while ${ }_{k} \Phi_{i, j}^{s}$ to ${ }_{k} \Phi_{i, j}^{s^{\prime}}$ should be completely different.

\section{LEAST SQUARES ESTIMATION}

Approximation (4) can be rewritten and used to get a constrained $\left(a_{k}^{s} \geq 0\right)$ set of linear equations which is established of:

$$
d_{t}=\left(\sum_{k}^{n} a_{k}^{s}{ }_{k} \Delta \Phi_{t}^{s}\right)-\left(\sum_{k}^{n^{\prime}} a_{k}^{s^{\prime}}{ }_{k} \Delta \Phi_{t}^{s^{\prime}}\right)-\Delta \Phi_{t}^{i n t} .
$$

Variable $t$ is an index of equations, $\Delta \Phi_{t}^{\text {int }}$ and $\Delta \Phi_{t}^{s}$ represent the measured unwrapped phase differences and the NWP simulated APS, respectively. This set of equations have to be minimized over the sum of $d_{t}$ which can be written as

$$
\begin{gathered}
\operatorname{argmin}_{a}\left\|A * a-\Delta \Phi^{i n t}\right\|_{2}, \text { while for every } \\
a_{i} \in a=\left(a_{1}, \ldots, a_{n},-a_{n+1}, \ldots,-a_{n+n^{\prime}}\right), a_{i} \geq 0
\end{gathered}
$$

and $A$ consists of $\Delta \Phi$ (see [3] for solving). Negative entries in $a$ follow from first minus in equation (5). Using the factors out of $a$, the restricted linear combination of the simulated APSs is the most plausible APS.

\section{ALGORITHM EXTENSION}

From a theoretical point of view, it is obvious that with the above minimization a deformation could be also reduced. But the question is, how probable and how big this behaviour is. To answer these questions, further investigations are necessary.

To make the algorithm more robust with respect to deformations, it can be extended. First of all, the sample space $i, j$ could be reduced onto a subspace where no deformation is expected at all. An estimation could be done with a sliding window implementation of this algorithm while monitor the coefficients. The total sum over $a$ should be near by 0 while most of the coefficients should be equal 0 .

Another approach is to extend the equation system, to two interferograms with one master scene temporally in the middle. If a linear deformation is assumed, the distortion should be at least lower because of the positive / negative deformation.

Another optimization is to monitor the coefficients and make them sparse. Coefficients near by 0 are less probable while absolute coefficients near by 1 are expected. Also, a time delay of 5 hours is much more unlikely than a time delay of 30 minutes. An iteration of minimisation, inspection and removing the corresponding APS is therefore an option. See the second example below for a first demonstration.

Of course practically, the restriction to an upper bound of the absolute coefficients is useful. This is demonstrated in both examples below.

\section{PERTURBATION}

For a first demonstration, this algorithm is applied to a real interferogram. The data are noisy and to make the demonstration more realistic, an artificial deformation is added (see figure 1). For the minimization, additional bounds for the coefficients $a_{i} \leq 1$ where applied and just the dry terms where used (for more details see [4]). The wet terms seems to be too chaotic to get good results. Three different NWPs with different micro physics where computed for the acquisition on 3 October, and two different NWPs for 4 October (a resolution of $1 \mathrm{~km}$ were used). Starting up from 21:00 UTC to 24:00 UTC with a 5 minute step size, the corresponding APSs were computed at both dates. That means 108, possible APS for 3 October and 72 possible APS for 4 October are generated.

The computed most plausible APSs are shown in figure $2(a, b)$. The difference between these two estimations is the introduced error, through the additional artificial deformation (see figure $2(\mathrm{c})$ ). In figure $3(\mathrm{a}, \mathrm{b})$, the APS corrected InSAR images are shown. As figure 3 (c) shows, the deformation is still in the data but unfortunately a little bit reduced (see figure 2 (c)). Figure 4 illustrates, that most of the coefficients are near by 0 . The selected NWPs, with coefficients below or equal -0.2 , reaches from 21:10 UTC to 21:40 UTC with a mean time delay of $\approx-17$ minutes (for the 4 October). Interestingly seven out of eight NWPs uses the same micro physics. Coefficients greater or equal 0.2 , reaches from 21:15 UTC to $22: 25$ UTC with a mean time delay of $\approx+15$ minutes (for the 3 October).

To demonstrate that the estimation bias of the artificial deformation can be reduced, a further restriction is made. The absolute value of the coefficients $a_{i}$ are considered as indicators for there importance. APSs were removed with coefficients near by 0 , the minimization is applied, and is repeated 


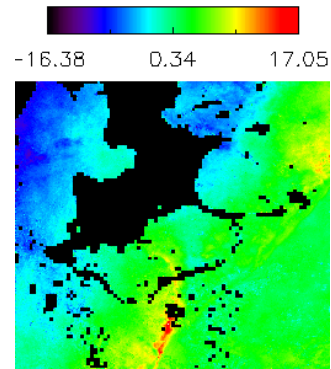

(a)

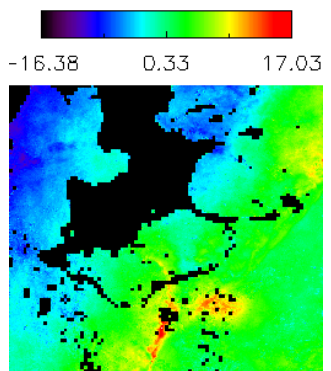

(b)

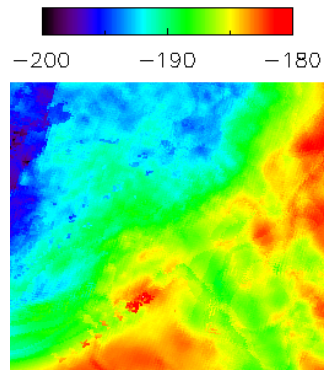

(a)

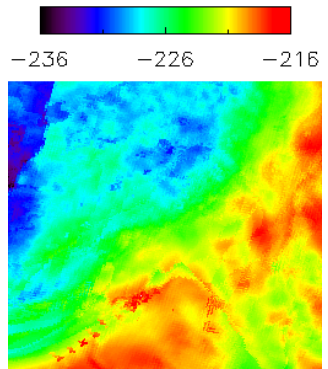

(b)

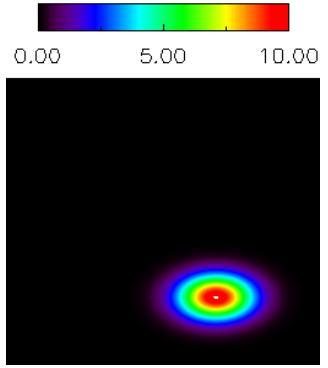

(c)

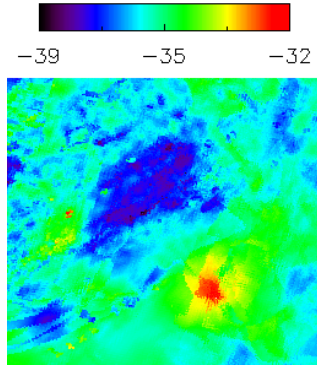

(c)
Fig. 1. (a) InSAR of 3 and 4 October 1995 at 21:41 UTC (see [5] for a detailed description) (b) InSAR + artificial deformation (c) Artificial deformation

until only two are left. In figure 5 (c) is shown that this time, the most plausible APSs just differ in the size of the coefficients. This means, there is no estimation bias of the artificial deformation, which is also shown in figure 6 (c).

\section{CONCLUSIONS}

A new approach for the APS mitigation bias has been developed and demonstrated. An ensemble of numerical weather predictions is utilized to estimate the most reasonable APS. The presented approach solves the problem of uncertainties in the NWP input data, the difficulty to fix physics options in the NWP (depending on the test site and weather situation) and NWP timing bias.

\section{REFERENCES}

[1] R. Holley, G. Wadge, and M. Zhu, Eds., New insights into the nature and effects of the water vapour field on InSAR measurements over Etna. FRINGE, 2007.

[2] B.M. Kampes, Radar interferometry: persistent scatterer technique, Remote sensing and digital image processing. Springer, 2006.

[3] Åke Björck, Numerical Methods for Least Squares Problems, SIAM, 1996.
Fig. 2. (a) Most plausible APS corresponding to InSAR (b) Most plausible APS corresponding to InSAR with artificial deformation (c) Difference between the two estimations (introduced error)

[4] J. L. Davis, T. A. Herring, I. I. Shapiro, A. E. E. Rogers, and G. Elgered, "Geodesy by radio interfereometry: Effects of atmospheric modeling errors on estimates of baseline length," Radio Science, vol. 20, pp. 1593-1607, 1985.

[5] R. F. Hanssen, T. M. Weckwerth, H. A. Zebker, and R. Klees, "High-resolution water vapor mapping from interferometric radar measurements," Science, vol. 283, no. 5406, pp. 1297-1299, 1999. 


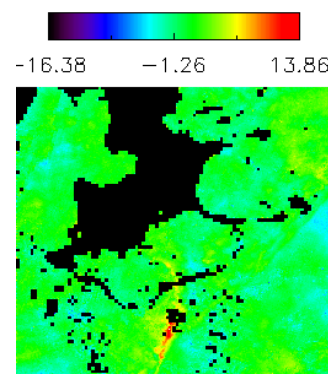

(a) (a)

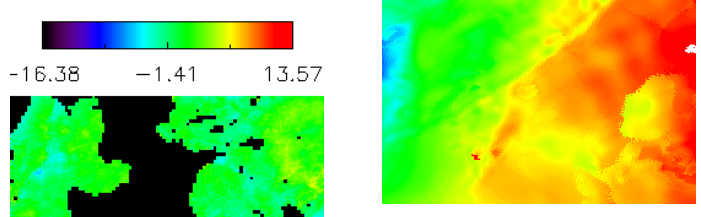

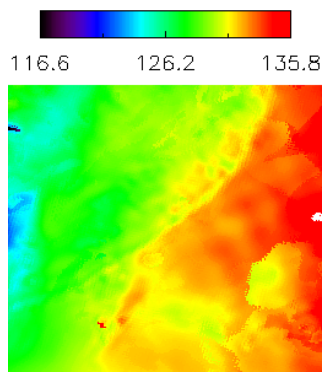

(b)

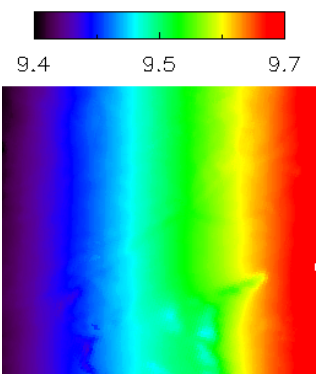

(c)

Fig. 5. (a) Most plausible APS corresponding to InSAR (sparsified) (b) Most plausible APS corresponding to InSAR with artificial deformation (sparsified) (c) Difference between the two estimations

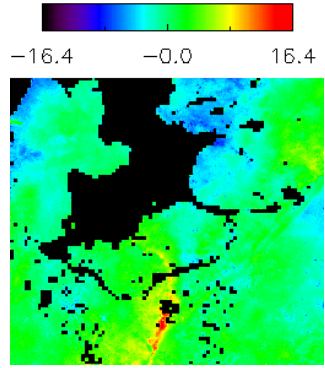

(a)

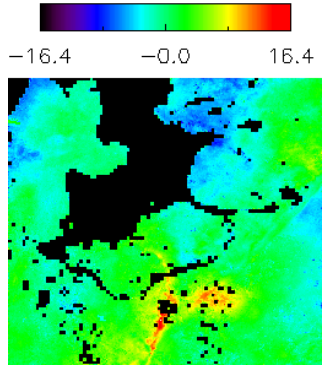

(b)

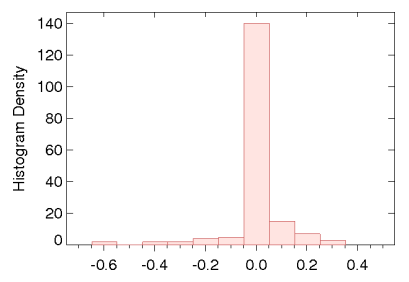

(a)

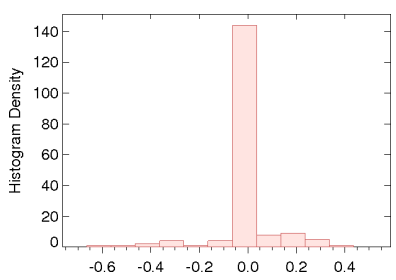

(b)
Fig. 4. Histograms of the computed coefficients corresponding to the estimations in figure 2 respectively

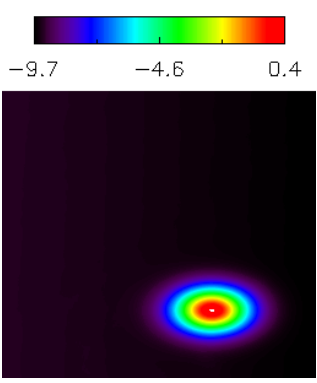

(c)

Fig. 6. (a, b) Differences between figure 1 and 5 respectively (c) Difference between the differences (fits nearly perfectly to the artificial deformation, so no error where introduced through the disturbance) 